\title{
La psicoconstrucción es innovación en el campo terapéutico
}

\author{
Dr. Fabiano de Abreu Agrela Rodrigues ${ }^{1}$ \\ deabreu.fabiano@gmail.com
}

\section{RESUMEN}

Comprender la mente humana en toda su complejidad es extremadamente desafiante. Los obstáculos para el estudio de algo que no es físicamente palpable son inmensos. Las terapias tradicionales son importantes para mapear la mente humana, pero el tratamiento se puede reconstruir a través de un método innovador que se basa en la memoria primitiva que está íntimamente ligada a la genética, a los ancestros. Se sabe que los mayores beneficios que conducen a la curación provienen de la información sobre los marcadores hereditarios. La psicoconstrucción modela funciones cognitivas, que llevan al sujeto a tomar decisiones y ajustar su rutina, de acuerdo a su singularidad.

Palabras clave: memoria; primaria; terapias; mente; síndromes; cerebro.

\footnotetext{
${ }^{1} \mathrm{PhD}$, neurocientista, mestre em psicanálise, biólogo, historiador, antropólogo, com formações também em neuropsicologia, neurolinguística, neuroplasticidade, inteligência artificial, neurociência aplicada à aprendizagem, filosofia, jornalismo, programação em python e formação profissional em nutrição clínica Diretor do Centro de Pesquisas e Análises Heráclito; Chefe do Departamento de Ciências e Tecnologia da Logos University International e da City University; Membro da SFN - Society for Neuroscience, Membro Redilat.
} 


\title{
Psychoconstruction is innovation in the therapeutic field
}

\begin{abstract}
Understanding the human mind in all its complexity is extremely difficult. The obstacles to the study of something that is not physically palpable are immense. Traditional therapies are important for mapping the human mind, but it can be reconstructed through an innovative method that is based on primitive memory that is closely linked to genetics, to ancestors. It is known that the greatest benefits that lead to healing come from information about genetic markers. Psychoconstruction models cognitive functions, which lead the subject to decision-making and adjustments in their routine, according to their uniqueness.
\end{abstract}

Keywords: memory; primary; therapies; mind; syndromes; brain

Artículo recibido: 02 enero 2022 Aceptado para publicación: 28 enero 2022 Correspondencia: deabreu.fabiano@gmail.com Conflictos de Interés: Ninguna que declarar 
Agrela Rodrigues...

\section{INTRODUCCIÓN}

La mente humana es poderosa: tiene 86 mil millones de neuronas y trillones de conexiones. A pesar de que el ser humano utiliza casi todo el potencial del cerebro, solo el $10 \%$ se utiliza para la parte cognitiva. La neurociencia se ocupa de permitir que las personas amplíen este sector de la capacidad cerebral. Tan misterioso y complejo.

Es en la etapa embrionaria que el ser humano comienza a formarse a través de la mielinización del sistema nervioso central; y acumulando memoria celular que te afectará tanto física como psicológicamente. El cerebro almacena información que se transmite de generación en generación, sin embargo, cada persona tiene su propio sello genético individual a modo de "huella dactilar", con vivencias únicas e imborrables.

La mente humana tiene una dimensión ilimitada y es un entramado de informaciones que existen desde la formación del ser humano, traídas en el ADN, pero también las que se van adquiriendo en cada momento de gestación, nacimiento y etapas del ciclo vital. Todo se almacena en el cerebro como una base de datos y algunos son de fácil acceso, otros son difíciles de conectar. Estos datos pueden establecer ramificaciones, cruzando información entre ellos; y a menudo están relacionados con la forma en que se adquieren y almacenan.

Para comprender la mente se construyó la idea de "arquitectura de la mente humana", a partir de la creación de cinco aspectos con base psicoanalítica: memoria primitiva, inconsciente, superinconsciente, preconsciente y consciente. La evolución de la inteligencia y el nivel de racionalidad está íntimamente ligada al almacenamiento de recuerdos, y la emoción circunstancial de ese momento, que interfiere mucho en la forma e intensidad del evento, para su posterior almacenamiento y evocación. Por tanto, cuanto más racional sea el ser humano, mejor afrontará los problemas, pues tendrá la capacidad de filtrar y eliminar lo que no le favorece.

Al poder comprender los tipos de recuerdos almacenados, es posible alcanzar un nivel superior en la comprensión íntima de los problemas psicológicos o psiquiátricos tanto de los demás como de nosotros mismos. Esto se debe a que ayudar a determinar dónde está la raíz del problema es fundamental y crucial. Cuando alguien es consciente del problema, su tipología y su localización, está a medio camino de aliviar el síndrome o trastorno y, en los casos en que sea posible, intentar un tratamiento. 
En este contexto, el uso de la racionalidad puede ser la forma de resolver trastornos o síndromes, la razón como filtro de la emoción. El paciente puede introspeccionar su problema utilizando técnicas para desarrollar la racionalidad aliada a un aumento en su autoconocimiento. En el caso de un problema psíquico, es necesario comprender los síntomas que generan los síndromes o trastornos que resultan en las acciones del día a día. Construyendo un patrón de aparición en el mundo, que atraviesa, por tanto, comportamiento e identidad fría. Un trauma, por ejemplo, puede pasarse por alto y la conciencia del paciente suaviza el trato. Las personalidades referentes al trastorno o síndrome pueden ser atendidas individualmente y comodidad para el paciente. Pero también es importante buscar consuelo y conciencia en una personalidad derivada de la memoria primitiva para una solución más determinada. Al estudiar las regiones del cerebro, se llega a la conclusión de que funcionan de manera interdependiente.

Con una ubicación determinada, nuestro cerebro emocional es primitivo, por lo tanto, es el primero en reaccionar instintivamente a las emociones y tratar de comportarse bajo el impacto inmediato que estas provocan. Sin embargo, toda la parte racional y más nueva del cerebro, en el piso superior de esta construcción, nos permite racionalizar el problema, asegurando así una mejor comprensión y una mejor solución para un resultado final más satisfactorio.

\section{TERAPIA TRADICIONAL}

Freud buscó incesantemente intelectualizar y racionalizar los traumas y miedos que estaban "grabados" en el inconsciente. Es como si estuvieran encriptados en la memoria más interna y/o primitiva y fueran accesibles a través de la relajación, una "meditación". El diván asume la simbología, el lugar donde la persona se concentraba y se enfocaba sólo en sí misma como "autohipnosis". Toda la atmósfera creada por él tenía un propósito. A través de palabras clave, impregnadas de importancia en la vida del sujeto, lo Simbólico dispara el recuerdo y la comprensión de los motivos ocultos dentro de uno mismo, por lo tanto, asociación libre, que vincula la información inconsciente para rastrear los motivos del comportamiento consciente.

En la interpretación de los sueños, la interpretación se lograba a través de los mensajes que se escondían en las imágenes. Freud creía que los sueños eran reveladores de emociones reprimidas, un medio para mostrarse subliminalmente. Con la elaboración secundaria del sueño, la persona organiza el pensamiento y el habla, lo que le permite 
comprender su propia confusión. El sueño es, en última instancia, un producto intrínseco del soñante, pero con la ayuda del analista, adquiere sentido y trascendencia y revela detalles que el paciente muchas veces es incapaz de discernir.

Lacan trabajó con símbolos, significantes y significados, toda la parte de la lingüística y no la energía libidinal como en Freud. Lenguaje corporal y verbal, así como lo no dicho. Delimitó la cronología temporal como determinante para la comprensión del paciente en sí mismo. Según su tiempo lógico, donde el paciente disponía de uno a: El momento de mirar; El tiempo de comprender; El momento de concluir. Porque ese tiempo se usó realmente para optimizar esta conclusión sobre sí mismo.

Las terapias permiten a las personas lidiar con problemas de comportamiento, para resolver problemas mentales y para el autoconocimiento. Las principales terapias tradicionales son:

\section{Psicoanálisis}

El psicoanálisis como teoría creada por Freud, tiene como objetivo primordial la cura por "escuchar y hablar", utilizado en casos de neurosis y psicosis. El método puede entenderse basado en la interpretación de los contenidos inconscientes de las palabras, acciones y producciones imaginarias del paciente. Esta interpretación la realiza el psicoanalista o analista, a partir de asociaciones libres y lo que se denomina transferencia, que sería la actualización de las emociones y sentimientos infantiles, ahora trasladados a la figura del analista y su comprensión real.

El método psicoanalítico es un procedimiento de arqueología del alma, en sus procesos mentales. Esta investigación busca pensamientos, sentimientos, emociones, fantasías y sueños a través del psicoanálisis del individuo. Se basa en la investigación de la persona, buscando comprender lo que es suyo, escondido de sí mismo. La terapia se adopta para tratar neurosis, histeria, psicosis y también enfermedades o problemas mentales. El método de investigación busca resaltar el significado inconsciente de las palabras, los deslices, las bromas, los sueños y las producciones imaginarias.

\section{Terapia de conducta cognitiva}

La Terapia Cognitivo-Conductual o TCC entiende la forma en que el ser humano interpreta los hechos como aquello que le afecta, y no los hechos en sí. En otras palabras: es la forma en que cada persona ve, siente y piensa sobre una situación que le provoca malestar, dolor, malestar, tristeza o cualquier otra sensación negativa. 
Es un enfoque terapéutico muy específico, claro y directo. Se utiliza para tratar varios trastornos mentales de manera eficiente. Su principal objetivo es identificar patrones de comportamiento, pensamiento, creencias limitantes y hábitos que están en el origen de los problemas, indicando, a partir de ello, técnicas para cambiar estas percepciones de forma positiva. La TCC está destinada tanto al tratamiento de diferentes trastornos psicológicos y emocionales como depresión, ansiedad, trastornos psicosomáticos, trastornos alimentarios, fobias, traumas, dependencia química, entre otros.

Y ayuda en las diversas cuestiones que involucran la vida en su conjunto: dificultades en las relaciones, elecciones profesionales, duelo, separaciones, pérdidas, estrés, dificultades de aprendizaje, desarrollo personal.

Sabiendo que el principal objetivo de la Terapia Cognitivo Conductual es cambiar los sistemas de significado de los pacientes para alterar sus emociones y comportamientos en relación con las situaciones, el primer paso de la terapia es comprender estos sistemas.

\section{Humanista existencial}

El enfoque Humanista Existencial es un método de investigación, descriptivo, centrado en la persona, y trata de la totalidad de cada uno en proceso de devenir, no es solo un estudio del ser humano, es un compromiso como deber humano .

El método debe ser fluido, permitiendo al individuo tener una perspectiva de su totalidad, desmitificando la idea de una realidad pura, confrontándola con otras realidades. La integración entre el individuo y el mundo le permite sentir la realidad presente, liberándose de las exigencias del pasado y del futuro. Contribuye a la autorrealización y ve al ser humano como sujeto activo y dueño de su realización.

El despertar al autodesarrollo puede darse a través del abordaje terapéutico humanista, con la ayuda del psicólogo. Éste debe actuar sin juzgar y sin encaminarlos a una estrategia sin antes hacer un enfrentamiento. El objetivo de la terapia humanista es proponer confrontaciones y cuestionamientos que lleven al individuo a reflexionar sobre su "yo ideal" y su autoimagen, buscando la congruencia, ya que es el individuo quien tiene el control del proceso terapéutico.

\section{Terapia Gestalt}

La Terapia Gestalt presta especial atención a la teoría de la percepción. La novedad de esta terapia es que tiene una finalidad terapéutica de ayudar a las personas que tienen alguna dificultad para ser felices. Pero este enfoque es recomendable para cualquier 
persona, independientemente de sus circunstancias, simplemente porque ofrece técnicas y recursos para tomar conciencia del propio potencial.

Gestalt es un enfoque psicoterapéutico centrado en el paciente, para que llegue a comprender lo que realmente está sucediendo en su vida ahora, en lugar de simplemente hablar sobre situaciones pasadas. A través del proceso de gestalt, los pacientes aprenden a ser más conscientes de sus propios patrones de pensamiento. Luego llegan a conocer comportamientos negativos que pueden bloquear la verdadera autoconciencia y hacerlos infelices.

Dentro de la terapia Gestalt, el paciente tiene espacio para explorar con seguridad sus experiencias sin miedo al juicio. De hecho, se alienta a los pacientes no solo a hablar sobre sus emociones o experiencias, sino a traerlas a la habitación. De esta manera, se pueden procesar en tiempo real con el terapeuta. Es decir, Gestalt es un enfoque que se enfoca más en la experiencia del cliente "aquí y ahora".

\section{Psicologia POSITIVA}

La Psicología Positiva emplea herramientas como la autorresponsabilidad para demostrar que todo el mundo tiene el poder de cambiar su realidad. Según este movimiento, el secreto para llevar una vida más satisfactoria es construir un modelo mental optimista, que siempre ve el "vaso medio lleno". Para ello, es necesario entrenar el cerebro y dejar de lado los patrones negativos, ejerciendo muchas veces mecanismos de gratitud y resiliencia.

El enfoque funciona desde construir y mejorar la calidad, centrándose en una perspectiva positiva para mantener la mente sana. Su práctica consiste en reconocer y ejercitar las emociones, las características individuales y las instituciones positivas, los tres pilares para lograr una vida más plena. Varias investigaciones científicas relacionan el bienestar mental con la prevención de enfermedades, ya que los estados emocionales negativos pueden contribuir al desarrollo o agravamiento de patologías.

La Psicología Positiva proporciona mayor satisfacción y felicidad en la vida, aportando motivación y energía para la consecución de objetivos. Buscan la superación y la entrega total a la tarea que realizan en el momento, disfrutando del aquí y ahora.

\section{Terapia Sistémica}

La terapia sistémica se centra en el análisis de las relaciones interpersonales que involucran a las personas y se basa en comprender los patrones de comunicación que se 
dan en el ámbito familiar, en el ámbito laboral o en las relaciones de pareja.

En otras palabras, se trata de estudiar a los individuos dentro de un sistema de relaciones.

De esta forma, para tratar una disfunción individual es importante iniciar una terapia de grupo.

Los especialistas en este enfoque terapéutico buscan patrones de comunicación que afectan negativamente a un colectivo (por ejemplo, una familia) y a través de la terapia corrigen estos patrones para que el paciente ya no tenga los síntomas que le causan angustia o sufrimiento.

En este enfoque terapéutico, la familia se concibe como un sistema, ya que los diferentes miembros que la componen crean interacciones emocionales y afectivas que determinan el comportamiento individual de cada miembro de la familia.

Este método ha demostrado ser efectivo para mejorar la socialización de las personas que padecen esquizofrenia, una condición que puede afectar dramáticamente el comportamiento. Desde un punto de vista sistémico se estudia al individuo con esta patología y se tiene en cuenta su entorno personal y familiar. En este sentido, psicólogos y psiquiatras recurren a la terapia familiar para mejorar la calidad de vida de los pacientes con esquizofrenia.

\section{PSICOCONSTRUCCIÓN}

La terapia de psicoconstrucción se basa en la memoria primitiva que está muy ligada a la genética, a los ancestros. El método de psicoconstrucción consiste, en primer lugar, en una consulta inicial, en la que el paciente puede decidir si quiere iniciar el proceso terapéutico a medio o largo plazo, o simplemente seguir lo que le indicaron en la consulta. Los mayores beneficios que conducen a la curación provienen de la información sobre los marcadores genéticos. En definitiva, se realiza la 'radiografía' del paciente, con sus rasgos de personalidad y perfil psicológico. Luego se define un horario con el cambio en su rutina.

La nueva terapia fue desarrollada en base a la genética. El ser humano puede llevar en sus células problemas desarrollados por los ancestros. Comienzan a solucionarlos sensibilizando sobre el problema, a partir de estrategias y pautas de resolución, ya sea de carácter psicológico o en las disfunciones provocadas por traumatismos, trastornos, síndromes y enfermedades. 
El método presenta al paciente técnicas y entrenamiento cognitivo para que pueda ganar autonomía y encontrar soluciones a problemas futuros, derivados de situaciones ya resueltas. Es decir, el patrón de comportamiento se encuentra a través de la observación de acciones pasadas, generadas por la forma en que el cerebro primitivo interpreta los estímulos externos. Es necesario identificar y modificar interpretaciones cristalizadas y disfuncionales. Esto hace que la persona se adapte mejor, se apropie de sí misma y, en consecuencia, sea más acogida, aceptada y feliz.

La psicoconstrucción dispone de numerosas técnicas que actúan en la raíz del problema. El método es una innovación dentro de los procesos terapéuticos ya utilizados. Basado en la neurociencia, la epigenética (un término que se refiere a los cambios en la actividad de los genes que no involucran la alteración del $\mathrm{ADN}$ ) se utiliza para construir un nuevo modelo, con un mejor desempeño psicológico y social.

La psicoconstrucción va más allá de la conciencia del trauma y no invalida las terapias psicoanalíticas. Sin embargo, suma datos genéticos en busca de la respuesta al por qué del trauma, cuál es el origen del trastorno, del síndrome o de cualquier problema que comprometa el equilibrio mental. La nueva terapia modela funciones cognitivas, que llevan al sujeto a tomar decisiones y ajustar su rutina, de acuerdo a su singularidad. El diseño del cerebro en su estructura nos permite “aprender a sentirnos mejor”, obteniendo un resultado más productivo, con respuestas más ajustadas a los estímulos del mundo exterior y una mejor comprensión de su entorno y contenidos internos.

- Un mismo trauma tiene diferentes respuestas según la personalidad del individuo, en relación a su memoria primitiva, a su genética.

\section{TERAPIA A TRAVÉS DE LA PSICOCONSTRUCCIÓN}

Es necesaria la concentración, la "meditación", una profundización a través de la racionalidad para encontrar la razón del problema en medio de la oscuridad del inconsciente. Activar la inteligencia emocional del individuo para que su región prefrontal sea determinante en la concentración y en la elección del contenido a buscar. El entrevistado encontrará en los engramas de sus recuerdos, hasta en los más mínimos rastros, las respuestas supuestas.

Tanto los conceptos de Freud como los de Lacan son coherentes para el proceso terapéutico, pero las respuestas pueden no ser ciertas. La realidad puede ser abstracta. 
Depositamos en nuestros engramas una memoria impresa determinada por la influencia de nuestra personalidad, nuestro cerebro reptiliano y paleolímbico.

En psicoconstrucción existen 5 pasos que determinan la intensidad del recuerdo en el engrama, por ejemplo, un vaso de agua y gotas de tinta negra, cada gota de tinta sugiere un recuerdo y los matices de emoción determinan la cantidad de gotas.

Imaginemos que cada vaso es una neurona, la claridad del agua en relación con la cantidad de gotas de tinta determina el grado de almacenamiento de ese recuerdo y esto está determinado por influencias emocionales que a su vez están determinadas por los matices de la personalidad. Buscar información en un vaso de agua clara puede inyectar gotas de tinta negra y oscurecer este vaso, dando la idea de que la información segura es la determinada. Pero la responsabilidad del presente puede determinar esta memoria y esta memoria no es real sino abstracta.

Así como la memoria almacenada puede haber sufrido ya un cambio de abstracción. Ya sea un recuerdo en el inconsciente, en el súper inconsciente o incluso en el preconsciente. El papel del terapeuta en estas condiciones también se vuelve intuitivo, obligándolo a ser una persona con una cognición muy desarrollada a través de su inteligencia, conocimiento y experiencia. Teniendo en cuenta que la información que trae el prefrontal puede haber sido creada o alterada, te obliga a retroceder en el tiempo, trayendo el conocimiento establecido en la ascendencia. "Efecto mariposa" factores en los que se crean posibilidades no permanentes en respuesta a una acción o actitudes, reales o inventadas. Evaluar en la condición de personalidad la relación de posibilidades, es decir, ese individuo pudo haber podido crear este recuerdo por influencia de su personalidad con interferencia del cerebro reptiliano.

En psicología y psicoanálisis tenemos la comprensión de las conductas, creamos razones basadas en la historia del paciente a través del trauma, la educación, factores relacionados con la vida consciente, buscando en el inconsciente la respuesta predeterminada por factores similares a la humanidad. La razón la encuentra aquel a quien la emoción le impide tomar la decisión y elección correctas; despejar el camino de una vida oscura ya que el otro no tiene la interferencia del yo, del cerebro reptiliano. Pero no solo están los matices del desarrollo desde el embrión hasta los 5 o 6 años de edad, sino también el factor genético en la memoria primitiva. 
El papel del psicoanalista no es sólo escuchar, sino también dejar hablar al paciente, ya que ese es el verdadero meollo del asunto. Al preparar tu discurso, también te escucharás a ti mismo, aportando tu razón e intelecto para organizar tus pensamientos y poner orden en tus sentimientos. Esta es una forma de fijar sus acciones en la vida, ponderar caminos y decisiones para adaptarse mejor y ser aceptado en la sociedad. Es la elaboración de mecanismos que interfieren positivamente en la producción de neurotransmisores que controlan nuestro estado de ser.

Comprender el por qué es diferente a encontrar una cura y, en este proyecto, mi concepto no determina una cura definitiva, sino continua. La cura sería en el sentido de un mayor bienestar ya que no existe una cura permanente para las marcas orgánicas. Las disfunciones en nuestros mensajeros químicos son irreversibles y están determinadas por factores, entre ellos: factores genéticos que influyen en el tiempo, el medio ambiente, en fin, detalles como micrómetros de un decimal periódico de sensaciones.

Mi proyecto revela una intención de terapia continua, determinada por tareas que van mucho más allá del conocimiento de la raíz del problema. Como mencioné anteriormente, el recuerdo puede estar dentro de una razón abstracta, conmovedora y convincente a sí misma. La asignación de tareas depende de la ascendencia del individuo, las posibilidades, las posibilidades genéticas, el trauma, los miedos, la inducción del cerebro reptiliano, la inteligencia emocional, la cognición y todos los demás rasgos de personalidad del paciente.

Con todo lo anterior, incluido en el protocolo, no solo podemos crear conductas sino hábitos que revelen un mayor bienestar a partir del tipo de alimentación, tareas, rutina, tareas que pueden alimentar el lado opuesto de lo que sujeta al individuo. una atmósfera que te hace daño.

Finalmente, me voy a usar como conejillo de indias. Propongo crear una tabla de personalidad de mi padre, mi madre, para desentrañarme. A partir de ahí pretendo llevar a cabo mi autoterapia para lograr una vida mejor. En última instancia, no busco ni pretendo curar. Buscamos una vida que fluya en paz, como los sofistas.

\section{ARQUITECTURA DEL CEREBRO}

El cerebro en su construcción de estructuras físicas con localizaciones concretas puede visualizarse en neuroimágenes y entenderse en su funcionalidad, de forma interdependiente. Su circuito neuronal permite conexiones en un sistema complejo y 
altamente dinámico. Pensamos en el cerebro sobre un eje cartesiano, separado por los hemisferios izquierdo y derecho.

Sin embargo, a partir de 1970, el neurocientífico estadounidense Paul MacLean acuñó el concepto de Triune Brain, una construcción evolutiva en tres plantas. Este modelo fue validado por toda la comunidad científica en la década de los 90 y se hizo muy utilizado por su coherencia neuroanatómica, contemplando una división evolutiva, que contiene 3 cerebros: el cerebro reptiliano, que es el sistema reptil (nuestra conciencia irracional); el cerebro límbico, que es el de los mamíferos, (nuestra conciencia emocional); y la neocorteza, que nos diferencia de todas las demás especies de la naturaleza (nuestra conciencia racional).

Todo el complejo reptiliano está dirigido principalmente a nuestra supervivencia, el Sistema Límbico se encarga de la interpretación y decodificación de las emociones y sentimientos que recibe del mundo externo con una base de datos del medio interno para una respuesta adaptativa, por lo tanto, el neocórtex es nuestro planta alta de esta psicoconstrucción, con funciones intelectuales superiores.

Y suma el neocórtex, es el área relacionada con la mente consciente; el límbico, al preconsciente; y el reptiliano, al inconsciente. Por tanto, utilizando la forma natural de construcción del cerebro, nuestra mente puede actuar, a través de la Terapia de Psicoconstrucción, de una forma más inteligente y adaptativa, aprendiendo a sentirse mejor.

\section{Cerebro reptiliano}

El cerebro reptiliano se encuentra debajo del campo temporal (hipocampo), cercano al esfenoides y también cercano a la amígdala, conocida como área reticular, esta región es la responsable de las primitivas acciones instintivas de supervivencia, ellas son: huir, luchar y congelarse. . Situado en lo más profundo del cerebro, siendo responsable de nuestra memoria primitiva, solo se activa cuando el peligro es evidente, es decir, la percepción de la vida y la muerte se vuelve amplia, por lo que el cerebro reptiliano tiene una estrategia utilizando la mente primitiva activando regiones fisiológicas. para maximizar los resultados positivos. Es del cerebro reptiliano que obtenemos la información esencial para activar reacciones primitivas, libera una cantidad extra de sangre a las regiones que se activan para la supervivencia, por ejemplo: piernas para correr, brazos para luchar. 
Agrela Rodrigues...

\section{Región límbica}

La segunda estructura cerebral que veremos es el cerebro paleolímbico, ubicado sobre el cerebro reptiliano y trae comportamientos en dos ejes: confianza en uno mismo y/o confianza en los demás. El paleo límbico es un cerebro reptiliano más rígido, específicamente trae consigo acciones como dominación, manipulación entre otras, ligadas directamente con los dos ejes. El cerebro neolímbico conforma la región del sistema límbico junto con el paleolímbico, pero a diferencia del paleolímbico, el neolímbico se encarga de nuestra conciencia, emociones, aprendizajes, gustos, motivaciones, entre otras cosas, el campo límbico se encarga de las personalidades: primaria (necesita recompensas para obtener emociones, sean positivas o negativas, conscientes o inconscientes); y secundaria (a diferencia de la primaria, está impulsada por lo que nos recuerda el entorno).

\section{Corteza prefrontal}

La corteza prefrontal, la última parte del mapeo de regiones cerebrales, es una evolución cerebral específica del ser humano, porque es grande, funciona como una computadora tomando información sensorial y creando nuevas perspectivas, ideas, teorías, sustrayendo del inconsciente y trabajar con esta información. Cuando el sistema límbico está en control, trae sensaciones como exclusión, negación, fracaso, como ya se explicó al principio, la corteza prefrontal debería ser la que mejor funcionaría con situaciones nuevas, pero cuando hay estos cambios, nuestro sistema límbico el cerebro está a mano adelante en el control del cerebro resistiendo nuevas perspectivas. Cuando logras cambiar de límbico a frontal, se acaba el estrés, surge la tranquilidad y aparecen nuevas inspiraciones, dejamos el piloto automático y pasamos las formas adaptativas, lo rígido se vuelve ligero, lo conflictivo se vuelve claro y objetivo.

\section{CASO}

Se trabajó el método de Psicoconstrucción con una paciente con HPD - Trastorno Histriónico de la Personalidad que sufrió prejuicios y rechazo por parte de la madre cuando era niña debido a que su piel era más oscura que la de sus hermanos. Esta información llevada a la conciencia del paciente, así como su causa, puede ayudar a encontrar consuelo a través de la conciencia y el cuidado de personalidades específicas. Pero el prejuicio de la madre cuando se transmite genéticamente al paciente hace que éste no se fortalezca del todo, ya que acaba prejuiciado contra sí mismo ya que el prejuicio 
está en su código genético. Por tanto, esta búsqueda en la memoria primitiva significa que también podemos ocuparnos del problema de raíz, que en este caso es el prejuicio.

En este caso concreto, la terapia partía de un tratamiento continuo, que abarcaba todos los mecanismos necesarios. Como se señaló anteriormente, el alivio del problema ocurre a través de la conciencia, pero no se resuelve si ya existen cicatrices resultantes de disfunciones trazadas por mensajeros químicos que controlan los sentimientos y las emociones. Además de concientización, conocimiento, estrategias para resolver cicatrices como el uso de neuroplasticidad cerebral, dieta, ejercicios físicos, conductas y hábitos acordes a la personalidad del paciente. También se realiza una prueba de ADN con riesgos genéticos para la salud y un análisis de sangre completo. En base a los resultados y con la ayuda de otros profesionales, perfilar todo lo necesario para que el paciente tenga la comodidad de llevar su vida de la mejor manera posible en un tratamiento que involucre todos los matices del problema, ya sea en la conciencia o en lo que él ya ha marcado, es decir, en la imposibilidad de desentrañar la realidad creada.

\section{CONCLUSIÓN}

Los seres humanos pueden llevar en sus células problemas desarrollados por antepasados y se sabe que los mayores beneficios que conducen a la curación se originan a partir de la información sobre marcadores genéticos. La terapia de psicoconstrucción se basa en la memoria primitiva que está íntimamente ligada a la genética, es una 'radiografía del alma' del paciente, con sus rasgos de personalidad y perfil psicológico. En la práctica, el paciente es sometido a la psicoconstrucción, en una reforma íntima que genera autonomía para encontrar soluciones a problemas futuros.

La psicoconstrucción basada en la neurociencia va más allá de la conciencia del trauma, no invalida las terapias psicoanalíticas y agrega datos genéticos que permiten al paciente tomar decisiones y ajustes para alcanzar el equilibrio mental. La responsabilidad de mantener la propia homeostasis trae al paciente cambios en su rutina de sueño, alimentación, actividad física, laboral e intelectual. Así como una mayor armonía en las relaciones, fomentando un modelo ecológico de salud y bienestar. La integralidad del cerebro al cuerpo, pasando por la comprensión de la mente y el conjunto de idiosincrasias que reverberan y expanden ondas concéntricas resultados de alcance exponencial según la participación, colaboración y voluntad de conquista del paciente y su madurez afectiva, 
emocional y de salud y física de la responsabilidad. El resultado es prometedor, ya que el cambio es de adentro hacia afuera. Entrar en la causa para cambiar los efectos.

\section{REFERENCIAS BIBLIOGRÁFICAS}

Luibil N, Patel BC. StatPearls [Internet]. Publicação StatPearls; Treasure Island (FL): 30 de março de 2019. Anatomia, cabeça e pescoço, órbita.

Kasai E, Kondo S, Kasai K. Variação morfológica na fossa craniana anterior. Clin Exp Dent Res. 2019 abr; 5 (2): 136-144.

Yamamoto M, Ho Cho $\mathrm{K}$, Murakami G, Abe S, Rodríguez-Vázquez JF. Desenvolvimento fetal precoce dos gânglios ótico e pterigopalatino, com referência especial à relação topográfica com o osso esfenoidal em desenvolvimento. Anat Rec (Hoboken). 2018 ago; 301 (8): 1442-1453.

Lopez-Serna R, Gomez-Amador JL, Barges-Coll J, Arriada-Mendicoa N, RomeroVargas S, Ramos-Peek M, Celis-Lopez MA, Revuelta-Gutierrez R, PortocarreroOrtiz L. Conhecimento da anatomia da base do crânio e implicações cirúrgicas do sacrifício humano entre as culturas

De Rosa A, Pineda J, Cavallo LM, Di Somma A, Romano A, Topczewski TE, Somma T, Solari D, Enseñat J, Cappabianca P, Prats-Galino A. Corredores endo- e extraorbitais endoscópicos para região esfeno-orbital : estudo anatômico com caso ilustrativo. Acta Neurochir (Viena). 2019

Craigie D. Elementos de anatomia geral, especial e comparativa. Edimburgo: Adam e Charles Black; 1838.

Comitê Federativo de Terminologia Anatômica. Terminologia anatomica. Estugarda: Thieme; 1998

Porter R. Dizionario Biografico da História da Medicina e da Ciência Natural. Milão: Franco Maria Ricci Ed; 1985

http://www.dacelulaaosistema.uff.br/?p=762

https://www.msdmanuals.com/pt-pt/casa/distúrbios-hormonais-emetabólicos/distúrbios-da-hipófise/considerações-gerais-sobre-a-hipófise https://www.news-medical.net/health/The-Limbic-System-and-Long-Term-Memory(Portuguese).aspx

https://siteantigo.portaleducacao.com.br/conteudo/artigos/educacao/que-estruturascerebrais-estao-envolvidas-nos-mecanismos-de-memoria/21575 
https://www1.folha.uol.com.br/ilustrissima/2019/11/o-que-a-ciencia-sabe-sobre-como-

memoria-e-guardada-e-transmitida.shtml mesoamericanas précolombianas. Foco em Neurocirurgia. 2012; 33 (2): E1. doi: 10.3171 / 2012.6.FOCUS 12120 